\title{
Surface textures of composite resins after combined wear test simulating both occlusal wear and brushing wear
}

\author{
Kiyoshi KAKUTA ${ }^{1}$, Amornrat WONGLAMSAM², Shin-ichi GOTO ${ }^{1}$ and Hideo OGURA ${ }^{1}$ \\ ${ }^{1}$ Department of Dental Materials Science, School of Life Dentistry at Niigata, The Nippon Dental University, 1-8 Hamaura-cho, Chuo-ku, Niigata \\ 951-8580, Japan \\ ${ }^{2}$ Faculty of Dentistry, Mahidol University, 6 Yothi St. Rajthevi, Bangkok 10400, Thailand \\ Corresponding author, Kiyoshi KAKUTA; E-mail: kakuta@ngt.ndu.ac.jp
}

\begin{abstract}
The purpose of this study was to investigate the relation between the texture of worn surfaces of composite resins and the maximum wear depth. Three types of composite resins were investigated: a hybrid composite resin consisting of irregular-shaped inorganic filler particles (APX); a composite resin which contained small, irregular-shaped, inorganic filler particles and large organic composite filler particles (SRE); and another which contained spherical inorganic filler particles and large organic composite filler (SDX). Surface profile measurement and elemental analysis were carried out on the worn surfaces of these three composite resins using an electron probe microanalyzer (EPMA). For the composite resin which exhibited the largest maximum wear depth, its surface texture was slightly rough with fine pores and grooves. For the composite resin with lowest maximum wear depth, it had a smooth worn surface due to the large organic composite filler being abraded during the combined wear test.
\end{abstract}

Keywords: Composite resin, Wear, Surface texture

\section{INTRODUCTION}

In dentistry, wear of composite resins is a clinical concern with performance-, reliability-, and longevity-related implications. Numerous studies have since been carried out to investigate the wear of composite resins ${ }^{1-3)}$, and most of them showed that wear is inevitable. In our recent study ${ }^{4}$ on wear of composite resins which contained large organic composite filler particles with nano-sized inorganic particles, they displayed markedly lower wear than other types of composite resins. In other words, apart from the hardness of materials, the wear of composite resins is also caused by other factors.

In this study, surface texture was the material factor to be investigated for its role and effect on the wear behavior of composite resins. Surface texture of a composite resin has considerable effect on the degree of surface friction against its antagonists. It was thus anticipated that an examination of the texture of a worn composite resin surface would give more insight into its wear mechanism.

Through elemental analysis and surface profile measurement, this study examined the relationship between the texture of worn surfaces of different types of composite resins and their depth of wear. Reasons for the lower wear of composite resins containing large organic composite filler with inorganic nanoparticles were also investigated.

\section{MATERIALS AND METHODS}

\section{Composite resins}

Three types of composite resins were investigated in this study. The first type was a hybrid composite resin consisting of irregular-shaped inorganic filler with different particle sizes (Clearfil AP-X, Lot No. 1149AA, Kuraray Medical Inc., Okayama, Japan; APX). The second composite resin consisted of irregular-shaped inorganic filler particles and large organic composite filler particles (Solare P, Lot No. 511082, GC Corp., Tokyo, Japan; SRE). The last composite resin consisted of spherical inorganic filler particles and large organic composite filler (Solidex-F, Lot No. 70610, Shofu Inc., Kyoto, Japan; SDX).

For SRE and SDX, the organic composite filler included inorganic nanoparticles in pre-cured resin.

\section{Specimen preparation}

Specimen preparation details were given in previous reports $^{4-6)}$. Briefly, each wear specimen was prepared using a metal mold (diameter: $15 \mathrm{~mm}$, depth: $8 \mathrm{~mm}$ ). Specimen thickness was adjusted to $2 \mathrm{~mm}$ using a spacer in the mold. Composite resin was filled into the mold and pressed with a glass slide to remove any excess. Overlapping irradiation was performed nine times, $40 \mathrm{~s}$ each time, through the glass slide using a curing light (Optilux 500, Kerr, Orange, CA, USA). Surface of the cured specimen was wet-polished using \#1000 SiC paper. Composite resin specimens with buffed surface finish were prepared to be used for comparison as buff-polished surface references.

\section{Combined wear test}

Details of the combined wear test were given in previous reports $^{4-6)}$. A combined wear test, which simulated occlusal wear and brushing wear alternately, was carried out on the surfaces of the composite resin specimens. In each wear cycle, occlusal wear was carried out 50 times followed by 20 times of brushing wear. For each specimen, this wear cycle was repeated 100 times for the 
Table 1 Combined wear test conditions used in this study

Combined wear test

Number of times in wear cycle:

Number of wear cycles:

Total number of times:

Occlusal wear

Antagonist material:

Antagonist configuration:

Occlusal force:

Sliding distance:

Loading and sliding cycle:

Medium:

Medium supply interval:

Brushing wear

Toothbrush:

Brushing force:

Sliding distance:

Brushing cycle:

Medium:

Slurry supply interval:
50 times of occlusal wear

20 times of brushing wear

100

5,000 times of occlusal wear

2,000 times of brushing wear

Aluminum nitride (HV 390)

Round tip (2 mm curvature)

$40 \mathrm{~N}$

$1 \mathrm{~mm}$

$1 \mathrm{~Hz}$

$5 \%$ cornstarch solution

Once per 50 times

Butler \#211, IL, USA

$1.5 \mathrm{~N}$

$30 \mathrm{~mm}$

$2 / 3 \mathrm{~Hz}$

Reference abrasive slurry (ISO 11609:1995)

Once per 20 times combined wear test. Table 1 shows the combined wear test conditions used in this study.

After the combined wear test, worn surface was washed with distilled water only to preserve surface condition. Using an ion sputter (JFC-1100, JEOL, Tokyo, Japan), worn surface was coated with gold for analysis by an electron probe microanalyzer (EPMA).

\section{Elemental analysis and surface profile measurement by EPMA}

Figure 1 shows a typical analysis area and roughness mean line used for the worn surfaces in this study. At 20 $\mathrm{kV}$ accelerating voltage and with wavelength dispersive X-ray spectroscopy (WDS), EPMA (JXA 8900, JEOL, Tokyo, Japan) was used for nondestructive elemental analysis and observation of surface topographic features (surface texture and roughness) in secondary electron images.

In our preliminary study, qualitative analysis for target elements of filler and resin components was performed. Results showed that carbon, silicon, aluminum, strontium, and barium were detected among the composite resins used in this study. In particular, oxygen, carbon, and silicon were detected in all the three composite resins. Carbon, an essential element of organic compounds, was also detected in high intensity as a resin component. Silicon, on the other hand, was detected in high intensity as an inorganic filler component. Distributions of carbon and silicon, and thus distributions of resin and inorganic filler components respectively, were indicated by color mappings: dark blue represented low intensity and red represented high intensity.

To compare surface roughness after the wear test, surface profiles of worn surfaces were measured using a 3D measuring microscope (STM6ZP, Olympus, Tokyo,

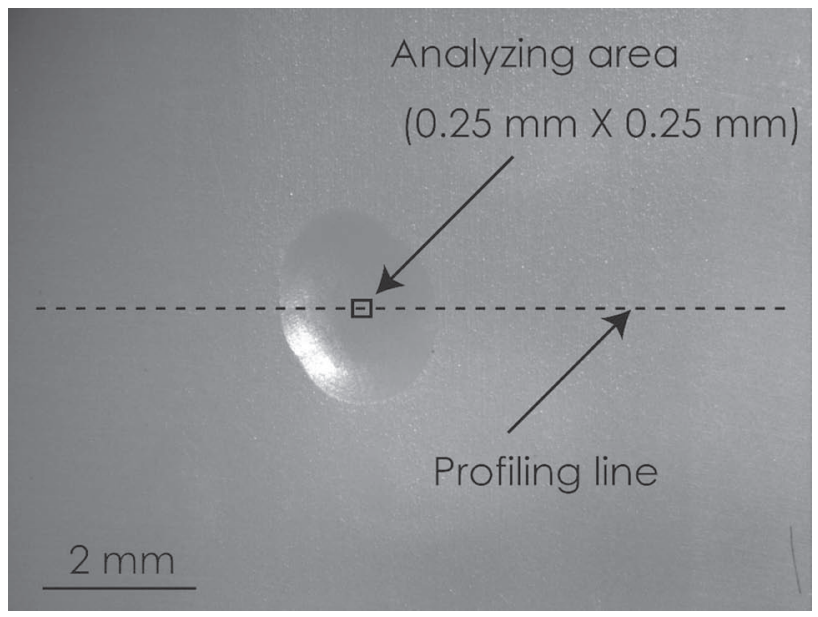

Fig. 1 Analysis area used for elemental analysis and roughness mean line used for surface profile measurement.

Japan). Surface textures of the worn surfaces were then observed through secondary electron images.

\section{RESULTS}

\section{APX composite resin}

1. Surface texture and distributions of carbon and silicon Figure 2 shows the secondary electron images and distributions of carbon and silicon on the surface of APX, which contained typical irregular-shaped inorganic hybrid filler. Image of the buff-polished surface showed many large irregular-shaped inorganic filler particles (A in Fig. 2). Carbon aggregated only in spaces between the large irregular-shaped inorganic filler, whereas silicon was detected in high intensity all over the buff-polished 


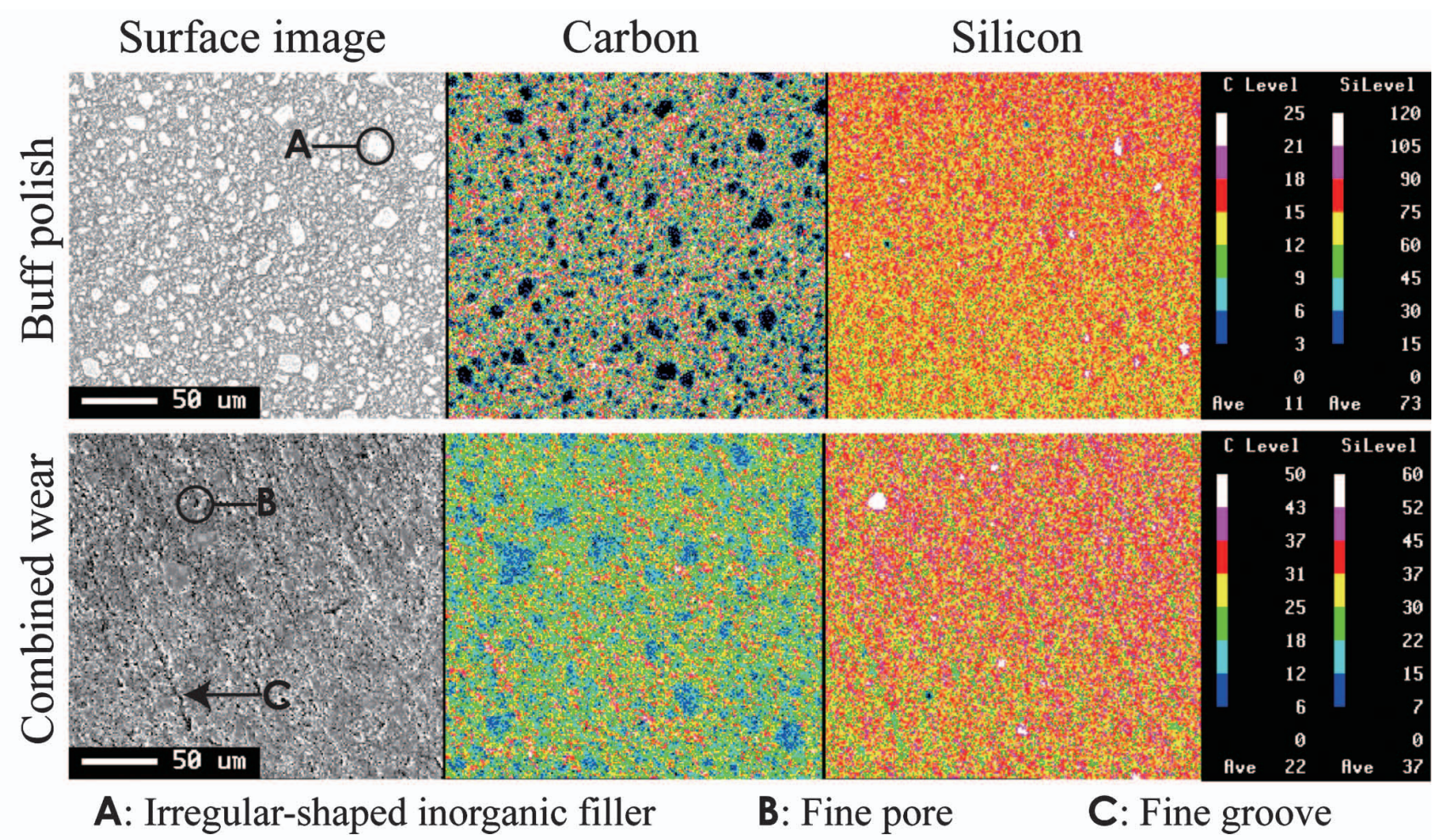

Fig. 2 Carbon and silicon distributions on the worn surface of APX and surface texture.

surface. This showed that APX surface consisted of large irregular-shaped inorganic filler and a mixture of resin and fine inorganic filler.

Texture of the combined test-worn surface of APX was different from that of buff-polished surface. After the wear test, APX revealed a flat worn surface with fine pores (B in Fig. 2) and grooves (C in Fig. 2). The large irregular-shaped inorganic filler particles clearly seen on the buff-polished surface were no longer easily recognized on the worn surface. Nonetheless, the blue colored areas in carbon distribution map indicated the presence of these large inorganic filler particles on the worn surface. A slight amount of carbon was detected on the large inorganic filler, whereas silicon was distributed all over the worn surface just like in the case of buff-polished surface. These images suggested that a mixture of resin and fine inorganic filler accumulated on the large irregular-shaped inorganic filler.

2. Surface profile

Figure 3 shows the surface profile of APX. Concavity in the profile indicated the combined test-worn area after occlusal wear and brushing wear were performed alternately. Toothbrush-worn area was identified near the concavity, which was an area where only the toothbrush was in contact with the composite resin surface during the combined wear test.

The profile showed that the combined wear test resulted in the highest wear. Nonetheless, surface roughness after the combined wear test was only slightly higher compared to that after \#1000 SiC polishing and

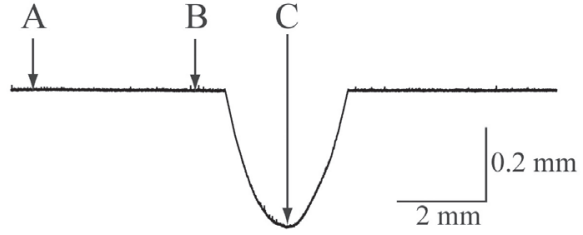

A: \#1000 SiC polished area

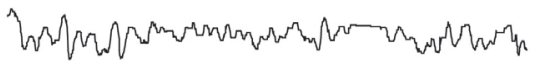

B: Toothbrush wear area

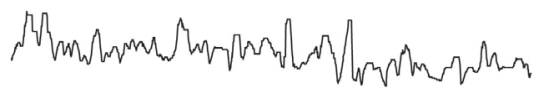

C: Combined wear area

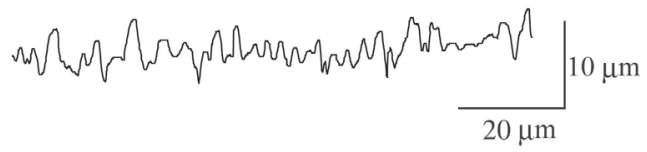

Fig. 3 Profile and roughness of APX surface.

quite similar to that after brushing wear, except that the peaks were slightly blunt compared to brushing wear. Among the composite resins tested in this study, APX showed the highest wear depth. 


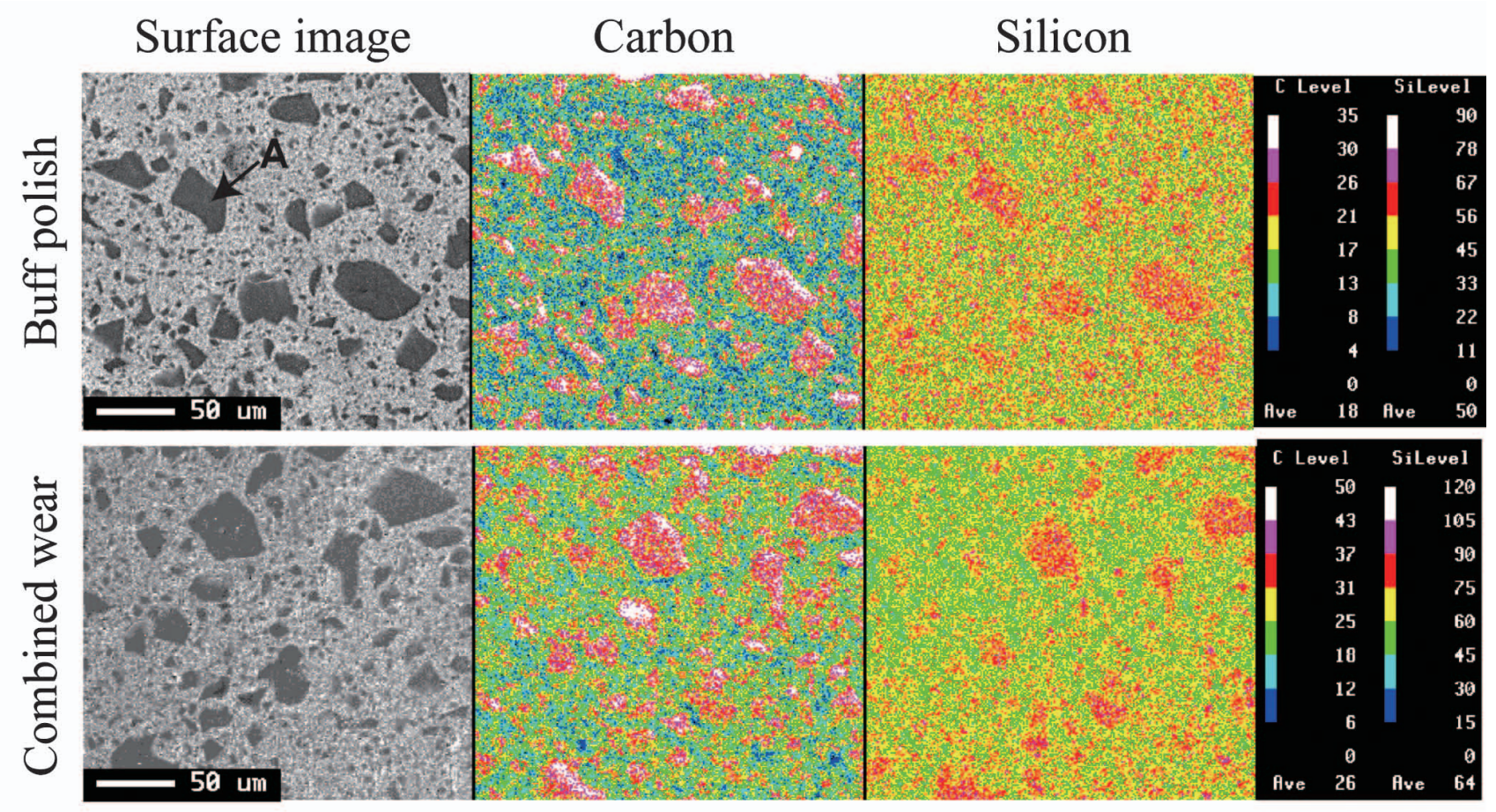

\section{A: Organic composite filler}

Fig. 4 Carbon and silicon distributions on the worn surface of SRE and surface texture.

\section{SRE composite resin}

1. Surface texture and distributions of carbon and silicon Figure 4 shows the secondary electron images and distributions of carbon and silicon on the surface of SRE, which contained irregular-shaped inorganic filler and organic composite filler. Large, flat, dark fragments (A in Fig. 4) were observed on the buff-polished surface. Large amounts of carbon and silicon were detected on these dark fragments, which were organic composite filler containing fine inorganic filler in pre-cured resin. Lower intensities of carbon and silicon were also detected in spaces between the organic composite filler. In these spaces, resin and relatively small irregular-shaped inorganic filler existed as a mixture. These results showed that SRE surface consisted of organic composite filler (which contained high densities of resin and fine inorganic filler) and a mixture of matrix resin and small inorganic filler.

Texture of the combined test-worn surface of SRE was the same as that of buff-polished surface. It was a smooth worn surface with flat dark fragments. In the spaces between the organic composite filler, carbon and silicon distribution maps showed that the intensity of carbon increased whereas that of silicon decreased. This suggested that during the combined wear test, the resin component accumulated on the worn surface of SRE.

2. Surface profile

Figure 5 shows the surface profile of SRE. Wear depth of SRE was smaller than that of APX. For SRE, surface roughness after the combined wear test was lower than

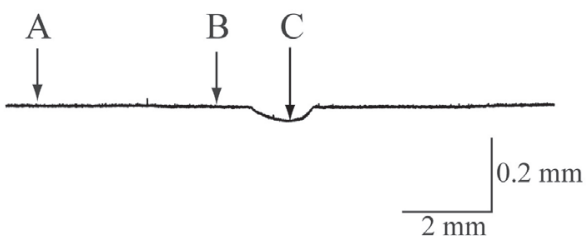

A: \#1000 SiC polished area

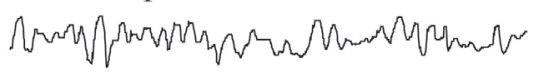

B: Toothbrush wear area

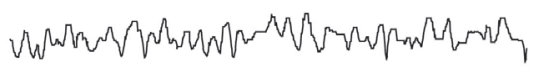

C: Combined wear area

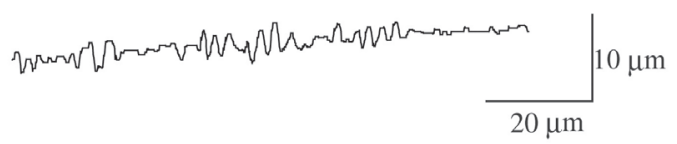

Fig. 5 Profile and roughness of SRE surface.

those after \#1000 SiC polishing and brushing wear. Both \#1000 SiC-polished surface and toothbrush-worn surface exhibited similar surface roughness. 


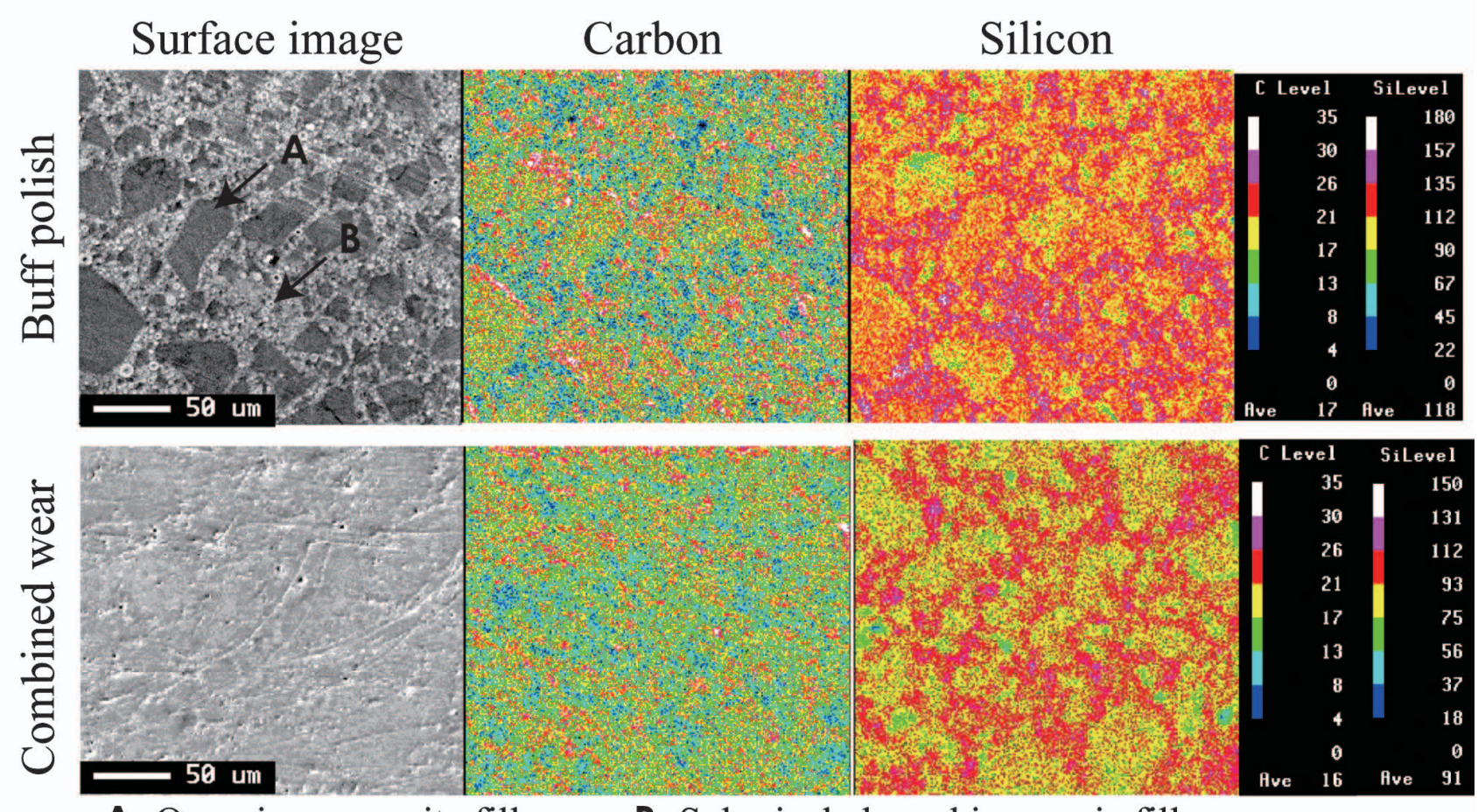

A: Organic composite filler

B: Spherical-shaped inorganic filler

Fig. 6 Carbon and silicon distributions on the worn surface of SDX and surface texture.

SDX composite resin

1. Surface texture and distributions of carbon and silicon Figure 6 shows the secondary electron images and distributions of carbon and silicon on the surface of SDX, which contained organic composite filler and sphericalshaped inorganic filler. Large dark fragments (A in Fig. 6) and small spherical-shaped filler (B in Fig. 6) were observed on the buff-polished surface. Carbon was detected mainly on the dark fragments, indicating that these dark fragments were organic composite filler particles. In the spaces between the organic composite filler, high intensities of silicon were detected. These results showed that SDX surface consisted of organic composite filler and a mixture of matrix resin and spherical-shaped inorganic filler, and that the latter mixture contained a high density of organic filler. For SDX, boundaries defining the distributions of components were not clear and distinct compared to the other composite resins.

Texture of the combined test-worn surface of SDX was different from that of buff-polished surface. On the buff-polished surface, the large dark fragments of organic composite filler were abraded by polishing. After the combined wear test, the organic composite filler and spherical-shaped inorganic filler could not be clearly distinguished. Surface image also revealed a homogeneous flat texture with a few scratches. Carbon and silicon distributions after the combined wear test were similar to those after buff polishing. However, boundaries of the organic composite filler became less

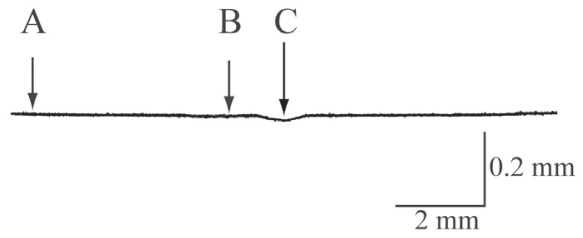

A: \#1000 SiC polished area

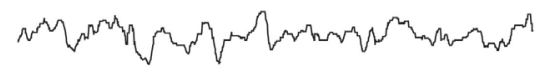

B: Toothbrush wear area

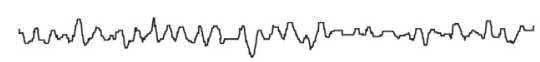

$\mathrm{C}$ : Combined wear area

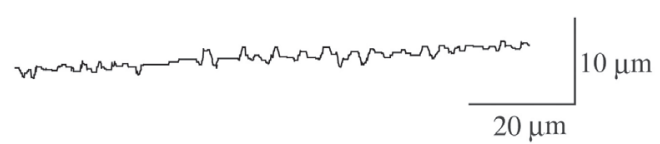

Fig. 7 Profile and roughness of SDX surface.

well defined after the combined wear test and silicon distribution also decreased slightly.

2. Surface profile

Figure 7 shows the surface profile of SDX. Among the 
composite resins tested in this study, SDX exhibited the smoothest profile after the combined wear test and hence the lowest wear depth. Brushing wear also delivered a smooth profile but deemed to be slightly rougher when compared to that after the combined wear test.

\section{DISCUSSION}

Effects of combined wear on the texture of worn surfaces In this study, the combined wear entailed both occlusal wear and brushing wear performed alternately on the same surface of a composite resin. The wear processes of both wear modes were quite different. In occlusal wear, surface abrasion and damage of the subsurface by the antagonist occurred at the contact area. At the same time, some abraded components accumulated on the worn surface to reduce friction between the worn surface and the antagonist.

During brushing wear, the toothbrush abraded the surface with fine abrasive particles in the medium and that only soft areas of the worn surface - such as the resin component- were abraded. This explained why the worn surface after brushing wear was slightly rougher than the worn surface after the combined wear test, as seen in Figs. 3, 5, and 7. Intraorally, wear of restorative material and teeth were caused under combined wear conditions.

In the combined wear test, alternation between the two wear modes rendered the worn surface more susceptible to wear. Rough surface caused by brushing wear increased friction between the worn surface and antagonist for every subsequent time of occlusal wear. Kon et al. ${ }^{6}$ ) showed that wear after a combined wear test tended to be higher than the sum of brushing wear and occlusal wear when performed singly.

Occlusal wear results in flat worn surfaces, while brushing wear results in rough surfaces. After the combined wear test in this study, texture of the worn surfaces was chiefly flattened surfaces produced by occlusal wear, as seen in Figs. 2, 4, and 6. In each wear cycle of the combined wear test, wear depth caused by occlusal wear was higher than the depth of roughness caused by brushing wear. Therefore, occlusal wear was the dominant wear process responsible for the texture of worn surfaces after the combined wear test in this study.

\section{Effects of filler system on the texture of worn surfaces}

During occlusal wear, the antagonist scratched away the worn surface. Some filler particles in composite resin were abraded from the worn surface, while others were compressed into the worn surface with components abraded by the antagonist. Therefore, abrasion and accumulation occurred at the same time on the worn surface during occlusal wear. This meant that filler behavior during the occlusal wear process was an important factor which affected the texture of worn surfaces.

1. APX composite resin

APX contained irregular-shaped inorganic filler and a mixture of resin and fine inorganic filler. As the irregular-shaped inorganic filler particles were hard and large, many voids were formed on the worn surface after they were abraded by the antagonist. At the same time, large irregular-shaped inorganic filler particles which remained on the worn surface were compressed into voids by the antagonist, resulting in fine pores and grooves (Fig. 2) and a slightly rough surface texture (Fig. $3)$.

The mixture of resin and fine inorganic filler of APX was also abraded and compressed by the antagonist. Carbon distribution in Fig. 2 showed that the worn surface, including areas occupied by the large irregularshaped inorganic filler, was covered with a small amount of resin component. Therefore, the compressed mixture of resin and fine inorganic filler was accumulated on the entire worn surface of APX. However, accumulation was not enough to fill in the fine pores and grooves around the large irregular-shaped inorganic filler.

2. SRE composite resin

SRE consisted of two phases: an organic composite filler and a mixture of inorganic filler and matrix resin. High intensities of carbon and silicon were detected on the organic composite filler, suggesting that a reinforced high-density resin and highly filled fine inorganic filler were used for the organic composite filler to improve strength. After the combined wear test, carbon and silicon distributions similar to those on buff-polished surface were observed. Surface image also suggested that the same smooth texture as the buff-polished surface was obtained. Surface profile in Fig. 5 further confirmed that SRE had a smooth worn surface after the combined wear test.

The smooth worn surface was a result of large organic composite filler being abraded by the antagonist during occlusal wear. It is conceivable that the organic composite filler of SRE was abraded more easily than inorganic filler of the same size, even if the organic composite filler consisted of a reinforced high-density resin and highly filled fine inorganic filler. Following abrasion, the abraded components accumulated on the worn surface, producing a thin mixture layer of resin and fine inorganic filler on the entire worn surface of SRE. An increase in carbon intensity and a decrease in silicon intensity in the spaces between the organic composite filler of the worn surface in Fig. 4 suggested that the thin mixture layer contained a high proportion of resin. Formation of the accumulated thin layer also accounted for the smooth surface texture of SRE after the combined wear test.

3. SDX composite resin

SDX contained organic composite filler and sphericalshaped inorganic filler. Figure 6 showed that a slightly high intensity of carbon was detected on the organic composite filler of the buff-polished surface, suggesting that a mildly high-density resin was used for the organic composite filler. On the other hand, a high intensity of silicon was detected in the spaces between the organic composite filler, suggesting that inorganic filler aggregated in the spaces between the organic composite filler. Therefore, the filler system of SDX was different 
from that of SRE although both composite resins contained organic composite fillers.

The surface profile of SDX in Fig. 7 showed the smoothest worn surface among the three composite resins tested in this study. Just like SRE, the smooth worn surface was a result of the large organic composite filler being abraded by the antagonist during occlusal wear. However, differences in their filler systems accounted for the difference in smoothness between SRE and SDX. Non-distinct boundaries in carbon and silicon distributions on the buff-polished surface in Fig. 6 suggested that mechanical properties did not vary sharply between the organic composite filler and matrix resin. Due to their similar mechanical properties, the antagonist abraded the composite resin surface uniformly during occlusal wear. Abraded components then accumulated on the worn surface, forming thereupon a mixture layer of resin and fine inorganic filler. The homogeneous texture of the worn surface and decrease in silicon intensity on the worn surface in Fig. 6 confirmed that the mixture layer was formed. Consequently, the homogeneous surface texture rendered SDX with the smoothest surface among the three composite resins tested in this study.

For SDX, its surface roughness caused by brushing wear (Fig. 7) was the lowest among the three composite resins. During brushing wear, the softer surface is selectively abraded by the toothbrush and fine abrasive particles in the medium. In the case of SDX, its surface was uniformly abraded. Therefore, the hardness of organic composite filler was similar to that of the resin matrix, with spherical-shaped inorganic filler around the organic composite filler. It was also anticipated that the surface of SDX was homogeneous not only in terms of roughness, but also in terms of hardness.

\section{Relation between surface texture and wear}

In a study by Wonglamsam et al. ${ }^{4}$, the same composite resins (i.e., APX, SRE, and SDX) were investigated. SRE and SDX were found to exhibit lower maximum wear depths (SRE: $0.08 \mathrm{~mm}$, SDX: $0.03 \mathrm{~mm}$ ) than APX (0.33 $\mathrm{mm})$. SRE and SDX had a common filler system namely, the organic composite filler. It was thus clear that the organic composite filler system affected the wear behavior of composite resins.

The worn surfaces of SRE and SDX had a smooth texture because the large organic composite filler particles were abraded by occlusal wear during the combined wear test. The smooth worn surface helped to reduce friction between the composite resin and antagonist during occlusal wear. Reduced friction then led to decreased wear of composite resin. For APX, its worn surface became flattened because of the accumulation of a mixture of resin and fine inorganic filler. However, presence of the remaining hard and large inorganic filler prevented the worn surface from becoming smooth. The hard and large inorganic filler in the worn surface induced subsurface damage when occlusal force was applied, resulting in a rough surface texture and material wear.

Material wear is generally said to be inversely proportional to hardness ${ }^{7}$. According to our data, the Vickers hardness values of APX, SRE, and SDX were 139, 58, and 48 respectively. Contrary to general thinking, APX exhibited the highest maximum wear depth whereas SDX exhibited the lowest. The contradiction between the maximum wear depth and hardness results was due to the composite material. Composite resins have a mixed structure consisting of soft matrix resin and hard filler particles, and this mixed structure complicates their wear behavior. In the case of APX, its hard and large inorganic filler might further aggravate surface wear. Results of this study suggested that instead of hardness, surface friction was a more important factor to the wear behavior of composite resins. Therefore, it is necessary to clarify the relationship between frictional forces and wear behavior of restorative materials in future studies.

\section{CONCLUSION}

Within the limitations of this study, the following conclusions were drawn:

1. Textures of the worn surfaces after the combined wear test differed among the composite resins.

2. The worn surface of APX, which exhibited the highest maximum wear depth, had a slightly rough surface texture with fine pores and grooves.

3. The worn surfaces of SRE and SDX, which exhibited low maximum wear depths, had a smooth surface texture because the organic composite filler particles were abraded by antagonist during occlusal wear.

4. Texture of the worn surfaces -more than hardness- affected the wear of composite resins.

\section{REFERENCES}

1) Powers JM, Ryan MD, Hosking DJ, Goldberg AJ. Comparison of in vitro and in vivo wear of composites. J Dent Res 1983; 62: 1089-1091

2) Shimizu T, Kitano T, Inoue M, Narikawa K, Fujii B. Ten-year longitudinal clinical evaluation of visible light cured posterior composite resin. Dent Mater J 1995; 14: 120-134.

3) Nagarajan VS, Jahanmir S, Thompson VP. In vitro contact wear of dental composites. Dent Mater 2004; 20: 63-71.

4) Wonglamsam A, Kakuta K, Ogura H. Effects of occlusal and brushing cycles on wear of composite resins in combined wear test. Dent Mater J 2008; 27: 243-250.

5) Kato K, Kakuta K, Ogura H. Effect of material and configuration of antagonist on combined wear. Dent Mater J 2005; 24: 368-376.

6) Kon M, Kakuta K, Ogura H. Effect of occlusal and brushing forces on wear of composite resins. Dent Mater J 2006; 25: 183-194.

7) Yamamoto Y, Kaneta S. Tribology. 1st ed. Tokyo: Rikogakusha Publishing; 2003. p. 188-190. 